\title{
Carnets
}

Revue électronique d'études françaises de l'APEF

Première Série - 3 Numéro Spécial | 2011

La littérature face au "politiquement correct». Notions, pratiques et dérives

\section{Les nouveaux Idéologismes}

Jacques De Decker

\section{(2) OpenEdition}

Journals

\section{Édition électronique}

URL : http://journals.openedition.org/carnets/6270

DOI : $10.4000 /$ carnets. 6270

ISSN : 1646-7698

Éditeur

APEF

Édition imprimée

Date de publication : 1 juin 2011

Pagination : 5-9

Référence électronique

Jacques De Decker, «Les nouveaux Idéologismes », Carnets [En ligne], Première Série - 3 Numéro

Spécial | 2011, mis en ligne le 19 juin 2018, consulté le 20 avril 2019. URL : http://

journals.openedition.org/carnets/6270; DOI : 10.4000/carnets.6270

\section{(c) (i) (9)}

Carnets est mis à disposition selon les termes de la licence Creative Commons - Atribution - Pas d'utilisation commerciale 4.0 International. 


\section{LES NOUVEAUX IDEOLOGISMES}

JACQUES DE DECKER

Secrétaire perpétuel de l'Académie royale de langue et littérature françaises de Belgique 
Les rebelles ont toujours abondé dans les œuvres littéraires ou théâtrales. Des figures diverses s'imposent à l'esprit: Antigone face à Créon, Hamlet face à Claudius, Sorel face à l'institution, Meursault face à la société toute entière. Ni les uns ni les autres, et tant d'autres encore, n'étaient alignés sur les idées en place. Antigone ne se pliait pas à la loi, Hamlet s'opposait au crime d'Etat, Sorel en voulait à une société inique, l'Etranger était tout simplement opposé au monde. ...

Ce qui nous pose question, c'est au contraire l'intrusion de l'acceptation du système non pas par les personnages fictifs, mais par les artistes eux-mêmes, sous couvert d'attitudes qui souvent miment le contraire. II ne s'agit pas des démarches artistiques qui avouent pleinement leur adhésion aux régimes sous lesquels elles se pratiquent. II y a des cas limites, certes, comme celui du réalisme socialiste, mais quels régimes n'ont pas connu leurs chantres? Racine est aussi l'homme de l'alignement face à un pouvoir monarchique et religieux, et cela n'enlève rien à son génie. De grandes œuvres, surtout plastiques et musicales, on les doit à des créateurs stipendiés et dociles - parfois tout au plus marginalement ironiques - face à l'autorité. Lorsque Michel Ange peint la Chapelle Sixtine, que le Caravage travaille sur commande des chevaliers de Malte, que Händel compose pour la cour d'Angleterre, ils ne se privent pas d'une part de contestation, mais veillent à ce qu'elle ne compromette pas la correction de l'ensemble. Nombreux sont, dans les œuvres classiques, les "dei ex machina" qui, en catastrophe, reviennent mettre l'église au milieu du village. Dans le "Tartuffe", cette conclusion intervient de justesse, mais avec suffisamment de maladresse pour que personne ne soit dupe. En d'autres termes, ces créateurs sont "politiquement corrects", tant dans leur acceptation des conditions générales dans lesquelles ils oeuvrent que dans leur souci de les critiquer non pas pour les abolir, mais les améliorer. II existe donc un "politiquement correct" positif, comme on parle du "bon" cholestérol.

Mais comment définir, dès lors, le mauvais "politiquement correct"? II s'agit d'une tartufferie, de positions qui ne sont plus authentiques, à savoir spontanées et sincères, mais soucieuses de se conformer à un "air du temps". Un air du temps qui se perçoit dans une société particulière, à savoir la nôtre, où il est bon de penser non pas selon ses convictions premières, mais de ce qu'il est recommandé d'adopter comme la norme. Non sans hypocrisie, souvent, parce que les opinions professées ne s'harmonisent pas avec le mode de vie, avec les intérêts réels. Les exemples grossiers sautent aux yeux.

Rien de plus confortable par exemple, que de favoriser les flux migratoires parce qu'ils sont sans incidence sur sa propre situation professionnelle, ou d'approuver une politique d'assistance généreuse quand on sait qu'on n'en aura pas besoin soi-même, bref, et c'est le résumé le plus simpliste, de penser à gauche alors qu'on se comporte à droite.

La gent intellectuelle emprunte le pas à cette tendance. C'est qu'elle part d'un présupposé erroné: celui qui pose que l'artiste, le penseur seraient préservés d'une inscription dans le 
système, qu'il bénéficierait, à l'intérieur de la société, d'une sorte d'extra-terrirorialité. Un artiste, en d'autres termes, ne saurait, par exemple, être bourgeois. Rubens l'était, Ingres l'était, mais depuis Picasso, même s'il est milliardaire, il ne peut plus l'être.

Dès lors, il pense non comme il est bien, mais comme il est beau de penser. Le paradoxe, dans la société moderne, c'est que les artistes de droite sont souvent des économiquement faibles, comme Céline, et ceux de gauche de bien nantis comme, à partir d'un certain moment de sa carrière, Aragon.

Le "politiquement correct", c'est l'adoption d'attitudes apparemment incorrectes (en fonction d'un ordre dominant postulé) pour des raisons d'opportunité esthétiques ou même, souvent, narcissiques. Une sorte de combinaison du confort de la vie courante et de l'inconfort apparent d'une pensée. Ce mal sévit particulièrement en France.

Au Portugal, une grande figure comme Pessoa a le mérite de ne pas tomber dans ce travers. II peut soutenir un pouvoir fort sans être un réactionnaire, admettre le capitalisme sans être le moins du monde à ses bottes. II est insituable, comme tous les génies. Qui sait ce que pensaient Shakespeare, ou Goethe, ou Tolstoï dans leur for intérieur? Ils étaient du côté de la vérité, de quelque bord qu'elle soit, dussent-ils pour cela se contredire ou décevoir une partie de leur audience. Le génie n'est pas celui qui est systématiquement pour ou contre, c'est celui qui est ailleurs...

Face à ces illustres exemples, on est en droit de se demander comment le "politiquement correct" a pu tant sévir, en France en particulier, et continuer de le faire. Le ton a été donné surtout après la deuxième guerre mondiale, époque où l'expression d' "intellectuel de gauche" a commencé d'être tenue pour un pléonasme, sottise que l'on a beaucoup entendue. Sartre a contribué à lancer le mouvement, avec des formules aussi sommaires que "le marxisme, philosophie incontournable" ou "il ne faut pas désespérer Billancourt". Les deux propositions sont des plus contestables. D'une part parce que le marxisme ne demeure vivant, et productif, que pour autant qu'il demeure discutable, amendable, adaptable, mais pas conservé dans le formol. D'autre part parce qu'il n'y a pas de pire insulte à la classe ouvrière que d'estimer qu'elle ne puisse affronter le désespoir, comme si elle était insuffisamment mûre pour au contraire le gérer, prendre appui dessus, en oeuvrant, par exemple, à améliorer le communisme, à le remettre sur la bonne voie.

Sur les raisons de l'attitude sartrienne, les hypothèses abondent. II y a celle de Dan Franck: Sartre se serait débondé dans la contestation à l'après-guerre parce qu'il se serait planqué durant celle-ci. II est vrai qu'il a très peu résisté. Fait prisonnier, il s'est évadé, puis s'est accommodé de l'occupation pour progresser dans sa carrière d'écrivain, notamment de dramaturge. Du coup, après le conflit, il aurait voulu se racheter. L'interprétation est un peu courte, partiale, et sans doute injuste, mais pas négligeable. 
II est très tentant de dire, dès lors, que le "politically correct" est né avec Sartre. II aura même son organe officiel, "Les Temps modernes". Sartre verra par exemple une dissidence se profiler, le "nouveau roman", et aura tôt fait de la récupérer avec sa préface à Nathalie Sarraute, et sa condamnation de Mauriac, qu'il désignera comme "romancier Dieu le Père", assimilation facile puisque l'auteur du "Désert de l'amour" était croyant. Or, Mauriac est l'un des rares à ne s'être pas égaré idéologiquement, en restant distant de Pétain et en repérant, comme Malraux, de De Gaulle était le bon cheval.

De Gaulle aussi était un élément majeur dans l'installation du "politically correct". II s'agissait d'être contre, à tous les coups. Et celui qui le respectait, l'admirait, était nécessairement un ringard, un moins que rien. D'où l'exclusion de Mauriac, déjà cité, de Malraux, de Romain Gary du champ des intellectuels dominants pendant des décennies. Les choses semblent actuellement s'améliorer pour Gary qui prend peu à peu sa place d'écrivain majeur de son temps, éclipsant en particulier le Nouveau Roman, ou pour Malraux, entré au Panthéon, mais pas pour Mauriac, dont il est toujours de bon ton de ne prendre en compte que le journaliste, au détriment de l'œuvre romanesque.

A cet égard, les travaux que vous me faites l'honneur d'ouvrir s'inscrivent dans le grand courant de la réévaluation de l'héritage du XXè siècle: il suffit de voir les noms des auteurs qui vont être évoqués.

Il y a, vous le voyez, du grain à moudre, mais l'organisation de cette journée est un très bon signe. Un signal de libération, d'émancipation à l'égard d'un amas d'idées reçues, dûment manufacturées par la bonne conscience, que j'appelle volontiers des "idéologismes". Déjà que je me méfie des idéologies, que je compare à des cancers, parce qu'elles sont faites d'idées mortes comme les cancers sont faits de cellules mortes. Les idéologies, pour changer de métaphore médicale, ce sont les caillots d'idéologie qui bouchent les canaux de la pensée. Ils sont là, bien arrimés dans nos artères intellectuelles, et ils empêchent les courants d'idées de passer. II faut les évacuer coûte que coûte. Ils sont des concentrés de "politically correctisme" solidifiés, bétonnés dans leur sûreté d'être du bon côté, de détenir le bon numéro. Ils sont, en fait, du prêt-à-porter de la réflexion, alors que chacun devrait réfléchir à sa propre mesure. C'est ce que font les écrivains évoqués en cette journée, c'est aussi le cas de Muray, de Nothomb, de Houellebecq, de Chessex, de Moix que j'aurais volontiers ajoutés au palmarès. Je connais un peu Nothomb, on ne se tutoie pas parce que dans son milieu cela ne se fait pas, mais je peux témoigner qui s'il est quelqu'un qui pense en parfaite non-alignée, c'est bien elle. Muray, j'ai un peu correspondu avec lui, je le suivais depuis vingt ans, et il est sûr que j'ai toujours estimé qu'il était un des esprits les plus déliés, les plus délivrés de son époque. J'ai rencontré Houellebecq et l'ai trouvé très vrai, très authentique, nourri d'une insurrection intérieure, tranquille, mais déterminée. Chessex était un ogre débonnaire, ultra-sympathique. Et Moix est un chien fou dont on aura encore 
beaucoup à attendre. Ils sont quelques repères des lettres françaises de notre temps, et je me devais donc de les citer à l'ouverture de ces débats. 
